\title{
Mini-jet thermalization and diffusion of transverse momentum correlation in high-energy heavy-ion collisions
}

\author{
Long-gang Pang, ${ }^{1}$ Qun Wang, ${ }^{1}$ Xin-Nian Wang, ${ }^{2,3}$ and Rong $\mathrm{Xu}^{4,3}$ \\ ${ }^{1}$ Interdisciplinary Center for Theoretical Study and Department of Modern Physics, \\ University of Science and Technology of China, Hefei 230026, China \\ ${ }^{2}$ Nuclear Science Division, MS 70R0319, Lawrence Berkeley National Laboratory, Berkeley, CA 94720 \\ ${ }^{3}$ Institut für Theoretische Physik, Johann Wolfgang Goethe-Universität, \\ Max-von-Laue-Str. 1, D-60438 Frankfurt am Main, Germany \\ ${ }^{4}$ Institute of Particle Physics, Huazhong Normal University, Wuhan 430079, China
}

\begin{abstract}
Transverse momentum correlation in azimuthal angle of produced hadrons due to mini-jets are studied first within the HIJING Monte Carlo model in high-energy heavy-ion collisions. Jet quenching in the early stage of thermalization is shown to lead to significant diffusion (broadening) of the correlation. Evolution of the transverse momentum density fluctuation that gives rise to such correlation in azimuthal angle in the later stage of heavy-ion collisions is further investigated within a linearized diffusion-like equation and is shown to be determined by the shear viscosity of the evolving dense matter. Such a diffusion equation for the transverse momentum fluctuation is solved with initial values given by HIJING and together with the hydrodynamic equation for the bulk medium. The final transverse momentum correlation in azimuthal angle is calculated along the freeze-out hyper-surface and is found further diffused for larger values of shear viscosity to entropy density ratio $\eta / s \sim 0.2-0.4$. Therefore the final transverse momentum correlation in azimuthal angle can be used to study the thermalization of mini-jets in the early stage of heavy-ion collisions and the viscous effect in the hydrodynamic evolution of the strongly coupled quark gluon plasma.
\end{abstract}

1. Introduction. In high-energy heavy-ion collisions at the Relativistic Heavy-ion Collider (RHIC), enormous amount of transverse energy is produced [1, 2, 3] via hard and semi-hard collisions of beam partons from each colliding nuclei [4, 5, [6, 7, 8]. Final state interaction among these produced partons during the early stage of heavy-ion collisions will lead to a thermalized (or partially thermalized) quark-gluon plasma (QGP) which will undergo further hydrodynamic evolution and expansion before hadronization and freeze-out. The strong elliptic flow as measured in non-central $A u+A u$ collisions at the RHIC energy [9] indicates indeed the early thermalization of the dense partonic system [10] before hydrodynamic expansion takes place and the momentum anisotropy of the medium partons is developed. The phenomenal jet quenching as observed in the suppression of large transverse momentum single hadron spectra [11] and back-to-back hadron correlation [12] also points to strong interaction between energetic partons and the bulk medium in the early stage of collisions. Extrapolation of the jet quenching picture to low and intermediate energy partons leads naturally to a picture of strong parton interaction in the bulk medium, a prerequisite for parton thermalization during the early stage of the formation of QGP in heavy-ion collisions.

Jets in high-energy collisions are characterized experimentally by a collimated distribution of hadrons in the direction of the underlying energetic parton and are measured as such in the calorimetric study of final transverse energy distribution. Such a calorimetric study of jets becomes problematic and eventually impossible when the transverse energy of jets becomes increasingly small and comparable to the background of the underlying soft interaction. However, the characteristic particle correla- tion within a jet and back-to-back correlation from di-jets should still be present in the azimuthal angle correlation of produced hadrons if mini-jets dominate the hadron production mechanism [13]. Such correlation due to jet and mini-jet production in heavy-ion collisions has been used to study jet quenching [12] and possible medium response to jet propagation [14] at RHIC. Extension of such study of correlation to the bulk matter should shed light on mini-jet thermalization throughout the evolution of the dense matter. Since correlations in a thermally equilibrated medium also carry information about the dynamics in an interacting QGP [15, 16, 17], study of the transverse momentum correlation in relativistic heavy ion collisions in principle can also provide constraints on the shear viscosity of the interacting dense medium [18, 19, 20]. Furthermore, experimental study of back-to-back hadron correlation in azimuthal angle can also provide information on the initial parton production mechanism and whether mono-jet production in the gluon saturation model [21] dominates.

In this paper, we propose and study the transverse momentum correlation in azimuthal angle as a direct measure of both the early parton thermalization and later viscous hydrodynamics evolution in high-energy heavyion collisions. We first calculate the transverse momentum correlation in azimuthal angle within the HIJING 22. Monte Carlo model and study the effect of early parton interaction through jet quenching mechanism. We will further study the evolution of transverse momentum fluctuation and correlation in azimuthal angle within the framework of viscous hydrodynamics and the diffusion of the transverse momentum correlation due to shear viscosity of the bulk matter.

2. Transverse momentum correlation. Instead of 
hadron multiplicity correlation, we will focus on the transverse momentum correlation for the purpose of our study, which should be less sensitive to the hadronization process and can be directly related to the momentum density fluctuation in an diffusion equation derived from viscous hydrodynamics [18, 19] as we will show later. We integrate over a finite size of psudo-rapidity $|\eta|<1.0$ and discretize the azimuthal angle $\phi$ into a number of bins whose size $\delta \phi \ll 1$ should be much smaller than the typical size of a jet. The transverse momentum correlation between two bins centered at azimuthal angle $\phi_{1}$ and $\phi_{2}$ is defined as

$$
C_{T}\left(\phi_{1}, \phi_{2}\right)=\frac{\left\langle\delta E_{T 1} \delta E_{T 2}\right\rangle}{\left\langle E_{T 1}\right\rangle\left\langle E_{T 2}\right\rangle},
$$

where $\delta E_{T j}=E_{T j}-\left\langle E_{T j}\right\rangle(j=1,2)$ are transverse momentum fluctuations in the $j$-th bin, $E_{T j}=$ $\sum_{i} p_{T i} \theta\left(\delta \phi / 2-\left|\phi_{i}-\phi_{j}\right|\right)$ are the total transverse momenta in each bin, and the average is taken over events.

We will first study the above transverse momentum correlation using the HIJING Monte Carlo model 22], which is based on a two-component description of soft and semi-hard interactions. Mini-jets contribute to a significant fraction of hadron production at the RHIC energy. Therefore, transverse momentum correlation $C_{T}$, as defined in Eq. (1), should reflect that of hadrons from mini-jets as shown in Fig. 1 as the dashed line for central $A u+A u$ collisions at the RHIC energy without jet quenching. The transverse momentum correlation is similar to two-hadron correlation [13] with finite transverse momentum cut-off. It contains both near-side correlation due to hadron production from a single mini-jet and backside correlation due to hadron production from two backto-back mini-jets. Final-state interaction in HIJING is modeled through a schematic parton energy loss mechanism, which will lead to a broadening or diffusion of the transverse momentum correlation as shown by the solid line in Fig. 1. Since the parton number density decreases very fast due to longitudinal expansion, mini-jet interaction is the strongest in the early stage and therefore jet quenching represents an early thermalization process for mini-jets in heavy-ion collisions. This will lead to significant modification of the characteristic correlation due to mini-jets. Therefore, the transverse momentum correlation in heavy-ion collisions can be used as a direct measure of mini-jet thermalization. Since the HIJING results without jet quenching is equivalent to that of a superposition of independent $p+p$ collisions, one therefore should compare heavy-ion collisions to $p+p$ to study the degree of mini-jet thermalization. As illustrated by the HIJING results in Fig. 1, early parton interaction leads to a significant diffusion of the near-side correlation while the shape of the away-side correlation remains almost intact since it is mainly controlled by the momentum conservation.

In non-central heavy-ion collisions, the transverse hydrodynamic expansion after the initial thermalization leads to a strong azimuthal asymmetry of hadron distri-
Figure 1: The transverse momentum correlation $C_{T}\left(\phi_{1}, \phi_{2}\right)$ from the HIJING Monte Carlo model in $A u+A u$ central collisions at $\sqrt{s}=200 \mathrm{GeV}$ with (solid) and without (dashed) jet quenching. In central collisions, $C_{T}\left(\phi_{1}, \phi_{2}\right)$ only depends on $\varphi=\phi_{1}-\phi_{2}$. The transverse momentum fluctuation in the same bin at $\varphi=0$ is subtracted from the correlation.

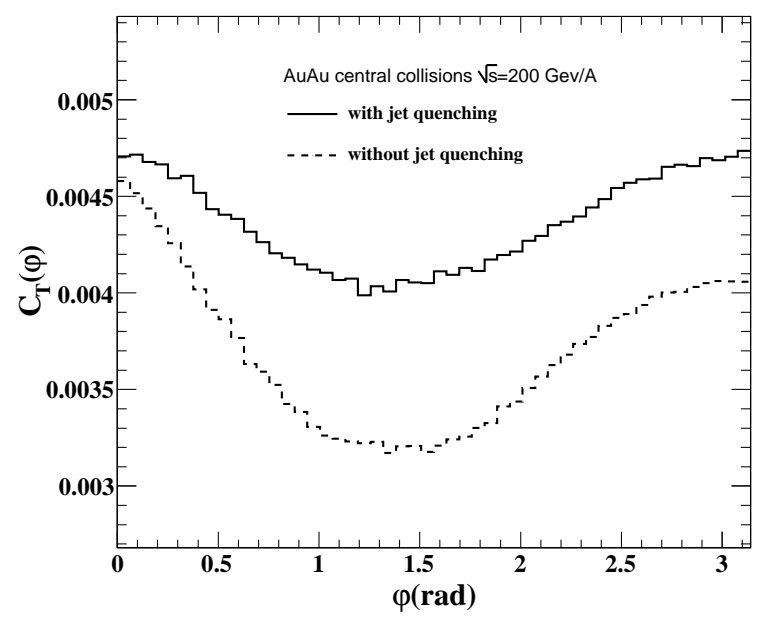

bution or elliptic flow [9, 23] induced by the asymmetry of the overlapping geometry and therefore will have similar two-hadron correlations as that in Fig. 1 due to minijets. But such correlation is for the averaged multiplicity and with respect to the nuclear reaction plane. This is different from the correlation in Eq. (1), which focuses on correlation of fluctuation from event to event. Nevertheless, there might exist contributions from elliptic flow to the transverse momentum correlation of our interest. Selection of central collisions will therefore help minimize the influence of the elliptic flow, which can be further reduced by averaging the transverse momentum correlation $C_{T}\left(\phi_{1}, \phi_{2}\right)$ over all possible pairs of bins with fixed width $\delta \phi$. In central collisions, $C_{T}$ only depends on $\varphi=\phi_{1}-\phi_{2}$, so we can use the notation $C_{T}(\varphi)=C_{T}\left(\phi_{1}-\phi_{2}\right)$.

3. Diffusion equation. After the initial (or partial) thermalization, we can assume that the system will obey the Bjorken scaling and can be described by the cylindrical coordinates $X^{\mu}=[\tau, \eta, r, \phi]$ with metrics $g_{\mu \nu}=$ $\operatorname{diag}\left(-1, \tau^{2}, 1, r^{2}\right)$, where $\tau \equiv \sqrt{t^{2}-z^{2}}$ is the proper time, $\eta$ the spatial rapidity, $r$ the transverse radius and $\phi$ the azimuthal angle in transverse plane. We will only consider the central rapidity region in central collisions and therefore the flow velocity can be cast in the simple form $u^{\mu}=\gamma_{T}\left[1,0, v_{T}, 0\right]$, where $\gamma_{T}=1 / \sqrt{1-v_{T}^{2}}$ and $v_{T}=v_{T}(\tau, r, \phi)$ is independent of the spatial rapidity $\eta$ in the Bjorken scaling scenario. Under this cylindrical system, we can neglect the diagonal components of the shear stress tensor $\pi^{r r}, \pi^{\tau \tau}$ and $\pi^{\phi \phi}$ as compared to their ideal counterpart $T_{0}^{r r}, T_{0}^{\tau \tau}$ and $T_{0}^{\phi \phi}$, and consider the only off-diagonal component $T^{\phi r}=\pi^{\phi r}=-\eta_{s} \gamma_{T}^{3} \partial_{\phi} v_{T} / r^{2}$ of the shear stress tensor in the $r$-component of the fluid 
equations, $\nabla_{\mu} T^{\mu r}=0$, which can be written as

$$
\begin{array}{r}
\frac{\eta_{s}}{r^{2}} \frac{\partial}{\partial \phi}\left(\gamma_{T}^{3} \frac{\partial v_{T}}{\partial \phi}\right)=\frac{1}{\tau} \frac{\partial}{\partial \tau}\left[\tau(\epsilon+P) \gamma_{T}^{2} v_{T}\right]+\frac{\partial P}{\partial r} \\
+\frac{1}{r}(\epsilon+P) \gamma_{T}^{2} v_{T}^{2}+\frac{\partial}{\partial r}\left[(\epsilon+P) \gamma_{T}^{2} v_{T}^{2}\right],
\end{array}
$$

where $\eta_{s}$ denotes the shear viscosity and should be independent of the azimuthal angle $\phi$ in the case of central heavy-ion collisions, and $\epsilon$ and $P$ are energy density and pressure, respectively.

As shown by the HIJING results in Fig. 1, the magnitude of the transverse momentum correlation due to mini-jets is quite small. Therefore, in the study of the evolution of the transverse momentum correlation, the fluctuation of energy-momentum density as a result of the correlation can be considered as a perturbation in a near-equilibrium system. Such a perturbation can be characterized by the perturbation $\delta v_{T}$ in the transverse flow velocity $v_{T} \rightarrow v_{T}+\delta v_{T}$. Neglecting fluctuation in $\epsilon$ and $P$ and considering central collisions where $v_{T}$ is independent of $\phi$, the equation for the corresponding fluctuation in the transverse flow velocity can be derived from Eq. (2) as,

$$
\begin{aligned}
\frac{\eta_{s}}{r^{2}} \gamma_{T}^{3} \frac{\partial^{2} \delta v_{T}}{\partial \phi^{2}}= & \frac{1}{\tau} \frac{\partial}{\partial \tau}\left[\tau(\epsilon+P) \gamma_{T}^{4}\left(1+v_{T}^{2}\right) \delta v_{T}\right] \\
& +\frac{2}{r} \frac{\partial}{\partial r}\left[r(\epsilon+P) \gamma_{T}^{4} v_{T} \delta v_{T}\right] .
\end{aligned}
$$

The above is a diffusion-like equation, which can be seen by observing that in the co-moving frame where $v_{T}=0$, the above equation becomes the standard diffusion equation $\partial\left(\tau \delta v_{T}\right) / \partial \tau=D \partial^{2}\left(\tau \delta v_{T}\right) / \partial \phi^{2}$ with the diffusion constant $D=\eta_{s} /\left(T s r^{2}\right)$ if one neglects the time-dependence of the enthalpy density $s T$.

With a given initial distribution of $\delta v_{T}$ one can solve the above diffusion equation and then calculate the final transverse energy fluctuation at the freeze-out surface,

$$
\begin{aligned}
\delta E_{T} & =\int d \Sigma_{\mu} u_{\nu} \delta T^{\nu \mu} \\
& =\Delta \eta \int\left(v_{T}-\frac{\partial \tau}{\partial r}\right) \gamma_{T} \delta g_{T} r \tau d r d \phi
\end{aligned}
$$

where $\Delta \eta$ is the spatial rapidity interval,

$$
d \Sigma_{\mu}=\left(-1,0, \frac{\partial \tau}{\partial r}, 0\right) r \tau d r d \phi d \eta
$$

is the freeze-out hyper-surface area element, and

$$
\delta g_{T}(\tau, r, \phi)=\gamma_{T}^{2}(\epsilon+P) \delta v_{T}
$$

is the fluctuation in transverse momentum density. One can then compute the azimuthal correlation of the transverse energy at freeze-out,

$$
\begin{aligned}
& C_{T}(\varphi)=\frac{(\Delta \eta)^{2}(\delta \phi)^{2}}{\left\langle E_{T}(\phi)\right\rangle_{\text {out }}^{2}} \int_{\text {out }} r_{1} \tau_{1} d r_{1} \int_{\text {out }} r_{2} \tau_{2} d r_{2} \\
& \times\left\langle\delta g_{T}\left(\tau_{1}\left(r_{1}\right), r_{1}, \phi\right) \delta g_{T}\left(\tau_{2}\left(r_{2}\right), r_{2}, \phi+\varphi\right)\right\rangle_{\text {out }}
\end{aligned}
$$

where the average should be over an ensemble of initial conditions for the spatial distribution of $\delta v_{T}$ and

$$
\begin{aligned}
\left\langle E_{T}\right\rangle_{\text {out }} & =\int_{\text {out }} d \Sigma_{\mu} u_{\nu} T^{\nu \mu} \\
& =\Delta \eta \delta \phi \int_{\text {out }}\left(1-v_{T} \frac{\partial \tau}{\partial r}\right) \gamma_{T} \epsilon r \tau d r .
\end{aligned}
$$

is the averaged transverse energy per bin.

To solve the diffusion equation (3), one has to provide the initial condition for $\delta v_{T}$ as well as the space-time evolution of $v_{T}, \epsilon$ and $P$ as functions of $\tau$ and $r$. To obtain the space-time dependence of $v_{T}, \epsilon$ and $P$, we solve the ideal hydrodynamic equations in $(2+1)$-dimension numerically [24, 25] with the initial transverse velocity $v_{T}\left(\tau_{0}\right)=0$ and initial temperature of $T=359 \mathrm{MeV}$ (corresponding to energy density $\epsilon_{0}=30.0 \mathrm{GeV} / \mathrm{fm}^{3}$ ) at the center of the overlap region of central $A u+A u$ collisions at an initial time $\tau_{0}=0.6 \mathrm{fm} / \mathrm{c}$. The initial transverse distributions of $\epsilon$ and $P$ are given by the Glauber model for nuclear collisions with the Woods-Saxon nuclear distribution. With such initial conditions for $(2+1)$ $\mathrm{d}$ ideal hydrodynamic equations, the final transverse energy $d E_{T} / d \eta=667 \mathrm{GeV}$, calculated at a freeze-out temperature $T_{f}=128 \mathrm{MeV}$ (or energy density 0.075 $\mathrm{GeV} / \mathrm{fm}^{3}$ ) in central (impact-parameter $\left.b=0\right) A u+A u$ collisions at $\sqrt{s}=200 \mathrm{GeV} / \mathrm{n}$, is consistent with the experimental value $d E_{T} / d \eta=606 \pm 35 \mathrm{GeV}$ for $0-5 \%$ central $A u+A u$ collisions [26], which is also close to the value of HIJING result $d E_{T} / d \eta=750 \mathrm{GeV}$. Note that the transverse energy as defined in Eq. (1), Eqs. (4)-(6) and (8) is slightly different from the calorimetric energy measured in experiments. However, the differences are small if one considers only hadrons in the central rapidity region.

We will use the HIJING model to estimate the initial condition for $\delta v_{T}$. We use the event ensemble for $\delta E_{T}$ generated by HIJING (with jet quenching) to extract the angular distribution for $\delta v_{T}\left(\tau_{0}\right)$ at the initial time according to Eqs. (4) and (6), assuming $\delta v_{T}\left(\tau_{0}\right)$ is independent of $r$ in the overlapped region for simplicity. This procedure of extracting the velocity fluctuation from HIJING, however, must rely on our knowledge of the initial values of energy density $\epsilon$ and pressure $P$ at the start of the hydrodynamic evolution when the system is in complete or partial thermalization at $\tau_{0}$. The initial values of energy density and pressure that we use for solving the ideal hydrodynamic equation, however, would give twice the total transverse energy from HIJING if we assume a free-streaming freeze-out at the initial time $\tau_{0}$. It is the $\mathrm{PV}$ work by the Bjorken longitudinal expansion in the $(2+1)$-d ideal hydrodynamics that causes the total transverse energy at the final freeze-out to be reduced by half. To match this initial condition for the ideal hydrodynamics, we will then multiply $\delta E_{T}$ from the HIJING events by a factor of 2 so that the transverse momentum correlation evaluated from Eqs. (4)-(8) with a free-streaming freeze-out at the initial time $\tau_{0}$ becomes identical to the HIJING result (with jet quenching) as shown in Fig. 1. 
Figure 2: (color online) The transverse momentum correlation evolved from the diffusion equation as a function of azimuthal angle difference $\varphi$ with different values of $\eta_{s} / s$ on the freezeout hyper-surface. The EOS is that with the first order phase transition or EOSQ.

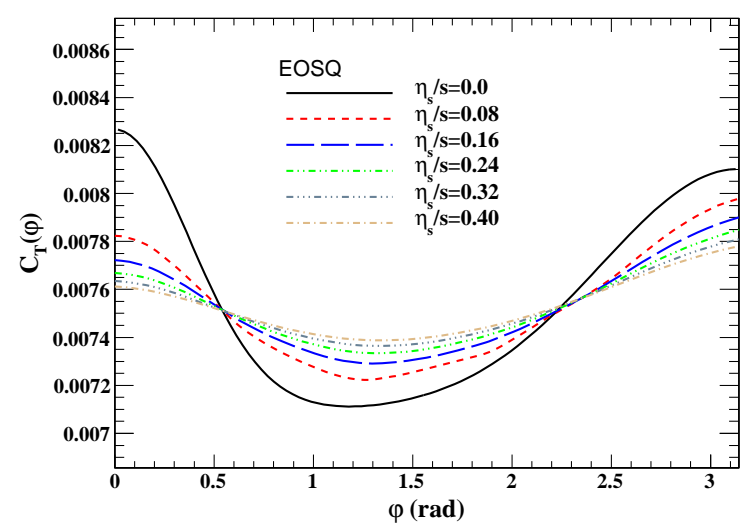

In the calculation of the transverse momentum correlation $C_{T}(\varphi)$ from HIJING events as shown in Fig. 1. we have removed the contribution from transverse momentum fluctuation $\sum_{i}\left\langle E_{T i}^{2}\right\rangle-\left\langle E_{T i}\right\rangle^{2}$ in the same bin at $\varphi=0$. Such fluctuation should also exist in the initial condition for $\delta v_{T}$ from HIJING events and will influence the transverse energy correlation at $\varphi \neq 0$ at a later time due to the diffusion according to Eq. (3). We will use the following procedure to remove the influence of the same-bin transverse momentum fluctuation on the final transverse momentum correlation through the diffusion equation. We first generate an event ensemble of $\delta v_{T}$ whose distribution in $\phi$ is random and the initial transverse momentum correlations all vanish except at the first bin $(\varphi=0)$. The magnitude of the random $\delta v_{T}$ distribution is adjusted such that the initial transverse correlation in the first bin at $\varphi=0$ matches that given by the HIJING events. We then solve the diffusion equation (3) with such a random distribution of $\delta v_{T}$ and calculate the event-averaged transverse momentum correlation $C_{T}^{(0)}(\varphi)$ at the same final freeze-out surface. Such correlation will be subtracted from the results with the HIJING initial condition to give us the final transverse energy correlation without the influence of the same-bin fluctuation.

Shown in Fig. 2 are the final event-averaged transverse momentum correlations $C_{T}(\varphi)$ from the solution of the diffusion equation (3) with different values of the shear viscosity to entropy density ratio $\eta_{s} / s$. The azimuthal dependence of $\delta g_{T}$ from the solution of Eq. (3) will be independent of time for zero viscosity $\eta_{s}=0$. However, its normalization will change because of the longitudinal and transverse expansion. During the evolution, both the transverse momentum fluctuation and the averaged transverse energy will decrease with time. However, the latter decreases faster as governed by the ideal hydrodynamics. This leads to the increase of the magnitude of the transverse correlation $C_{T}(\varphi)$ due to hydrodynamic expansion even for ideal fluid with $\eta_{s} / s=0$ while the shape of the correlation remains the same. This is consistent with the observation that the magnitude of the transverse energy correlation from HIJING with jet quenching is larger than that without as shown in Fig. 1.

As one can see, the transverse momentum correlation becomes diffused as one increases the value of shear viscosity to entropy density ratio, which characterizes the momentum exchange among nearby fluid elements in different bins of azimuthal angle. This is consistent with the HIJING results with and without jet quenching since jet quenching happens at the earliest stage of heavy-ion collisions during the initial thermalization process where the shear viscosity is the largest. The diffusion of the correlation in the near-side due to the viscous evolution is much stronger than the away-side. We have also studied the dependence of the final transverse momentum correlation on the equation of state (EOS). We find that the shape of the final correlation is not very sensitive to the EOS, so we present in Fig. 2 only the result of the EOS with the first order phase transition or EOSQ.

4. Conclusions and discussions. We have studied transverse energy correlation in azimuthal angle and its evolution in high-energy heavy-ion collisions. We show that such correlation will have a characteristic structure of two Gaussian peaks in high-energy heavy-ion collisions at the RHIC energy and above due to the dominance of mini-jets in the initial parton production. We find that within the HIJING Monte Carlo model, the transverse energy correlation will be significantly broadened or diffused by jet quenching in the early stage of thermalization in heavy-ion collisions. Assuming that the residual correlation still exists in the nearly-equilibrated system, we derived a diffusion-like equation for the local transverse energy density fluctuation by taking linearized approximation of the viscous fluid equations. With the initial condition as given by the HIJING results, we solved such a diffusion equation in combination with the space-time evolution of the averaged thermodynamic variables and flow velocity as given by the ideal hydrodynamic equations. The final transverse energy correlation of produced hadrons averaged over many events is found to be diffused because of the shear viscosity. For larger values of the shear viscosity to entropy density ratio, $\eta_{s} / s \sim 0.2-0.4$, the diffusion is as significant as that caused by jet quenching. One can therefore essentially study the degree of mini-jet thermalization in heavy-ion collisions as well as the viscous effect of the strongly coupled QGP [27, 28] by measuring the transverse momentum correlation in heavy-ion collisions in comparison with that in $p+p$ and $p+A$.

The correlations of transverse momentum per particle in both pseudo-rapidity and azimuthal angle have been measured by the STAR experiment [29] at RHIC through an autocorrelation technique which inverts the bin-size dependence of the event-wise mean transverse momentum fluctuation to extract the bin-bin correlation. 
Such correlation is different from the correlation of the transverse momentum which is the total transverse momentum within a bin with given size $\delta \phi$. Nevertheless the transverse momentum correlation that is inferred in the STAR experiments and its centrality dependence 29] might point to medium modification of the jet fragmentation and hadronization process that could also influence the transverse momentum correlation as studied in this paper. Such effect beyond the normal jet hadronization and hydrodynamic evolution of the medium will be interesting to investigate through experimental measurement of the transverse momentum correlation as proposed in this paper.

We thank Y. F. Wu for insightful discussions that got us interested in this problem, and U. Heinz for providing the code for solving the ideal hydrodynamic equations. We also thank J. Dunlop and L. Ray for discussions on experimental measurements of transverse momentum correlation. This work is supported by NSFC of China under Projects No. 10825523, No. 10675109 and No. 10735040 and MOE of China under Project No. IRT0624, and by the Director, Office of Energy Research, Office of High Energy and Nuclear Physics, Divisions of Nuclear Physics, of the U.S. Department of Energy under Contract No. DE-AC02-05CH11231. Q. Wang is supported in part by '100 talents' project of CAS. During the completion of this work, R. Xu was supported in part by the Helmholtz International Center for FAIR within the framework of the LOEWE program (Landes-Offensive zur Entwicklung Wissenschaftlich-ökonomischer Exzellenz) launched by the State of Hesse, Germany. X.-N. Wang thanks the hospitality of the Institut für Theoretische Physik, Johann Wolfgang Goethe-Universität and support by the ExtreMe Matter Institute EMMI in the framework of the Helmholtz Alliance Program of the Helmholtz Association (HA216/EMMI) during the completion of this work.
[1] B. B. Back et al. [PHOBOS Collaboration], Phys. Rev. Lett. 85, 3100 (2000) arXiv:hep-ex/0007036.

[2] K. Adcox et al. [PHENIX Collaboration], Phys. Rev. Lett. 87, 052301 (2001).

[3] C. Adler et al. [STAR Collaboration], Phys. Rev. Lett. 87, 112303 (2001) arXiv:nucl-ex/0106004.

[4] R. C. Hwa and K. Kajantie, Phys. Rev. Lett. 56, 696 (1986).

[5] K. Kajantie, P. V. Landshoff and J. Lindfors, Phys. Rev. Lett. 59, 2527 (1987).

[6] K. J. Eskola, K. Kajantie and J. Lindfors, Nucl. Phys. B 323, 37 (1989).

[7] X. N. Wang and M. Gyulassy, Phys. Rev. Lett. 68, 1480 (1992).

[8] K. J. Eskola, B. Muller and X. N. Wang, Phys. Lett. B 374, 20 (1996) arXiv:hep-ph/9509285.

[9] K. H. Ackermann et al. [STAR Collaboration], Phys. Rev. Lett. 86, 402 (2001).

[10] U. W. Heinz, AIP Conf. Proc. 739, 163 (2005).

[11] K. Adcox et al. [PHENIX Collaboration], Phys. Rev. Lett. 88, 022301 (2002).

[12] C. Adler et al. [STAR Collaboration], Phys. Rev. Lett. 90, 082302 (2003).

[13] X. N. Wang, Phys. Rev. D 46, 1900 (1992); D 47, 2754 (1993) .

[14] S. S. Adler et al. [PHENIX Collaboration], Phys. Rev. Lett. 97, 052301 (2006).

[15] M. A. Stephanov, K. Rajagopal and E. V. Shuryak, Phys.
Rev. D 60, 114028 (1999).

[16] S. Jeon and V. Koch, Phys. Rev. Lett. 85, 2076 (2000).

[17] M. A. Stephanov, Phys. Rev. D 65, 096008 (2002).

[18] M. A. Aziz and S. Gavin, Phys. Rev. C 70, 034905 (2004).

[19] S. Gavin and M. Abdel-Aziz, Phys. Rev. Lett. 97, 162302 (2006).

[20] M. Wang, L. Li, L. Liu and Y. Wu, J. Phys. G 36, 064070 (2009).

[21] D. Kharzeev, E. Levin and L. McLerran, Nucl. Phys. A 748, 627 (2005).

[22] X. N. Wang and M. Gyulassy, Phys. Rev. D 44, 3501 (1991); M. Gyulassy and X. N. Wang, Comput. Phys. Commun. 83, 307 (1994).

[23] J. Y. Ollitrault, Phys. Rev. D 46, 229 (1992).

[24] P. F. Kolb, J. Sollfrank and U. W. Heinz, Phys. Rev. C 62, 054909 (2000) arXiv:hep-ph/0006129.

[25] P. F. Kolb and U. W. Heinz, arXiv:nucl-th/0305084

[26] S. S. Adler et al. [PHENIX Collaboration], Phys. Rev. C 71, 034908 (2005) [Erratum-ibid. C 71, 049901 (2005)] arXiv:nucl-ex/0409015.

[27] M. Gyulassy and L. McLerran, Nucl. Phys. A 750, 30 (2005).

[28] G. Policastro, D. T. Son and A. O. Starinets, Phys. Rev. Lett. 87, 081601 (2001) arXiv:hep-th/0104066.

[29] J. Adams et al. [STAR Collaboration], J. Phys. G 32, L37 (2006) arXiv:nucl-ex/0509030. 